\title{
Application Value of Slider-Crank Mechanism in Pick-and-Place Operation of Delta Robot
}

\author{
QIN Zhe ${ }^{1,2}$, LIU Xiao-chu ${ }^{1,2}$, ZHAO Zhuan ${ }^{1,2}$, XIAO Jin-rui ${ }^{1,2}$ \\ ${ }^{1}$ Guangzhou University, School of Mechanical and Electric Engineering, Guangzhou 510006; \\ ${ }^{2}$ Key Laboratory of High-Performance Metal Materials Reinforced Grinding Machining, Guangzhou 510006
}

\begin{abstract}
By absorbing the advantages of the rotary-driven Delta robot and linear-driven Delta robot, a Delta robot for pick-and-place operation that forms a crank-slider at the drive joint is designed.To take the most common gate shaped curve in Cartesian space as the motion trail of robotic pick-and-place operation, according to the kinematics inverse solution theory of Delta robot, this thesis mainly solves the output angular velocity of robot-driven joint. Establishing the static transfer mathematical model and solving the forced condition of driving joint. The simulation analysis show that after the upper slider-crank mechanism is connected to the driving joint, the angular velocity of the driving joint changes suddenly, which caused a rigid impact on the robot in the picking and releasing operation, though the force of the driving joint can be made smaller.
\end{abstract}

\section{Introduction}

Delta robots have been proposed by Dr. CLAVEL since 1985[1] and many prototypes for different purposes have been made. Most of these robot widely apply used in electronics, food, medicine and other fields with the advantages of high bearing capacity, high positioning accuracy, simple structure, easy to achieve high-speed movement, etc[2-6]. Many research works have been developed the Delta series of robots include: Pierrot team delvelop H4 I4, Heli4 and Par4 and so on[7-9] in which industrial acceleration reaches $12 \mathrm{~g}$ and experimental acceleration reaches $20 \mathrm{~g}$ of Par4. Adept company developed a four degree of freedom high-speed SCARA parallel robot based on the prototype[10] of Par4 robot. And the robot claims to be the fastest in the world. Huang Tian[11] simplified Delta mechanism as Planar mechanism Diamond and invented TJU-Delta manipulator.

At present, to further improve the accuracy, stability and stiffness of the high-speed, high-frequency and pick-and-place operation, the present ways cover two aspects[12-13]: reducing the inertia of moving parts by lightweight design, improving the dynamic performance index by scale integrated; reducing the deviation between the actual pose and the pose of the command using high speed and high precision control system. Improve the precision, stability and rigidity of Delta robot's high-speed, high-frequency and pick-and-place operation play an important role in the design stage and running process.
This article by absorbing the advantages of the rotary-driven Delta robot and linear-driven Delta robot[14-15], a Delta robot for pick-and-place operation that forms a crank-slider at the drive joint is designed.To take the most common gate shaped curve in Cartesian space as the motion trail of robotic pick-and-place operation, according to the kinematics inverse solution theory of Delta robot, this thesis mainly solves the output angular velocity of robot-driven joint, the output force and torque of robot-driven joint, the output linear displacement of the slider that linked at the drive joints. Finally, analyze the value of the Slider-Crank Mechanism used for Pick-and-Place Operation of Delta Robot.

\section{Solve the angular velocity of the drive joint}

\subsection{Trajectory planning for pick-and-place}

The pick-and-place operation consists of three parts, including vertical, horizontal and vertical, as shown in Fig.1. The robot's ending part moves from point $\mathrm{A}$ at the time of $\mathrm{t}=0$, completes the entire pick-and-place operation at point $\mathrm{D}$, and divides the whole motion process from $\mathrm{A}$ to $\mathrm{D}$ into $\mathrm{N}$ parts according to time shown in equation (1) Sequence, which is analyzing the position coordinates, velocity and acceleration about the robot's ending part relative to the time variable t[16].

$$
t_{i}=T \cdot \frac{i}{N}(i=0,1, \cdots, N)
$$




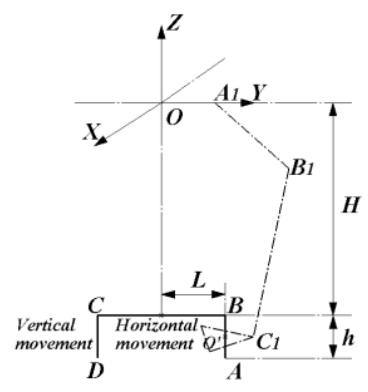

Figure 1. Pick and place operation track

(1) The robot moves along the vertical axis from point A to point $\mathrm{B}$ when $0 \leq S_{A B}\left(t_{i}\right) \leq h$, during this process, the position coordinates, velocity and acceleration about $t_{i}$ are as follows (2)

$$
\left\{\begin{array}{c}
\boldsymbol{S}_{A B}=\left(\begin{array}{lll}
0 & L / 2 & -S_{A B}\left(t_{i}\right)-H
\end{array}\right)^{T} \\
\boldsymbol{V}_{A B}=\left(\begin{array}{lll}
0 & 0 & -S_{A B}^{\prime}\left(t_{i}\right)
\end{array}\right)^{T} \\
\boldsymbol{A}_{A B}=\left(\begin{array}{lll}
0 & 0 & -S_{A B}^{\prime \prime}\left(t_{i}\right)
\end{array}\right)^{T}
\end{array}\right.
$$

(2) When $-\mathrm{L} / 2 \leq S_{B C}\left(t_{i}\right) \leq \mathrm{L} / 2$, the robot's ending part moves from point $\mathrm{B}$ to point $\mathrm{C}$ in the horizontal direction. At this time, the motion path height $\mathrm{z}$ remains unchanged, the position coordinates, velocity and acceleration about $t_{i}$ are as follows (3)

$$
\left\{\begin{array}{l}
\boldsymbol{S}_{\boldsymbol{B C}}=\left(\begin{array}{lll}
0 & S_{B C}\left(t_{i}\right) & -H
\end{array}\right)^{T} \\
\boldsymbol{V}_{\boldsymbol{B C}}=\left(\begin{array}{lll}
0 & S_{B C}^{\prime}\left(t_{i}\right) & 0
\end{array}\right)^{T} \\
\boldsymbol{A}_{\boldsymbol{B C}}=\left(\begin{array}{lll}
0 & S_{B C}^{\prime \prime}\left(t_{i}\right) & 0
\end{array}\right)^{T}
\end{array}\right.
$$

(3) Finally, the robot's ending part moves from point $\mathrm{C}$ down to point $\mathrm{D}$ along the vertical axis to complete the entire pick-up process when $0 \leq S_{C D}\left(t_{i}\right) \leq h$. At this time, the position coordinates, velocity and acceleraion about $t_{i}$ are as follows (4)

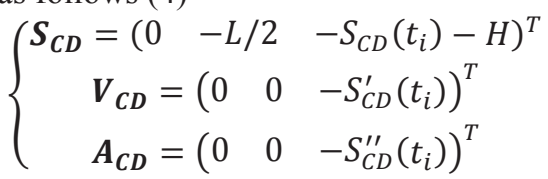

Through the above equations (2) (4), you can ensure the position coordinates $\boldsymbol{S}$, speed $\boldsymbol{V}$, acceleration $\boldsymbol{A}$ that are relative to the changing of $\mathrm{t}$ about the robot's ending part movement in the entire pick-up cycle. Fig.2.

The sketch diagram of Delta mechanism, as shown in

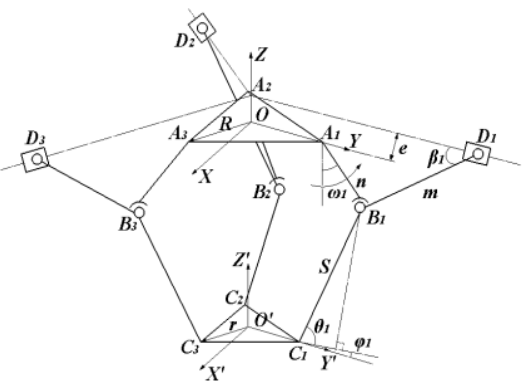

Figure 2. Sketch diagram of Delta mechanism

After solving the motion position of coordinate $S$ about the robot's ending part in the base, the angular velocity of the drive joint can be solved by using the Delta parallel mechanism inverse theory, as the following equation $(5) \sim(8)$

$\left(r+y-n \sin \omega_{1}-R\right)^{2}+\left(z+n \cos \omega_{1}\right)^{2}=S^{2}$

$$
\begin{array}{r}
\left(\frac{\sqrt{3}}{2} R-\frac{\sqrt{3}}{2} r+\frac{\sqrt{3}}{2} n \sin \omega_{2}\right)^{2}+\left(z+n \cos \omega_{2}\right)^{2}+ \\
\left(y-\frac{1}{2} r+\frac{1}{2} n \sin \omega_{2}+\frac{1}{2} R\right)^{2}=S^{2}(6) \\
\left(x+\frac{\sqrt{3}}{2} r-\frac{\sqrt{3}}{2} n \sin \omega_{3}-\frac{\sqrt{3}}{2} R\right)^{2}+\left(z+n \cos \omega_{3}\right)^{2}+ \\
\left(y-\frac{1}{2} r+\frac{1}{2} n \sin \omega_{3}+\frac{1}{2} R\right)^{2}=S^{2} \\
{\left[\begin{array}{lll}
x_{A B} & y_{A B} & z_{A B} \\
x_{B C} & y_{B C} & z_{B C} \\
x_{C D} & y_{C D} & z_{C D}
\end{array}\right]=\left[\begin{array}{ccc}
0 & L / 2 & -S_{A B}\left(t_{i}\right)-H \\
0 & S_{B C}\left(t_{i}\right) & -H \\
0 & -L / 2 & -S_{C D}\left(t_{i}\right)-H
\end{array}\right]}
\end{array}
$$

\subsection{Solution the angular velocity of during the pick-and-place operation}

The corresponding parameters of Delta parallel mechanism was shown in Tab.1.

Table1 Delta mechanism parameters

\begin{tabular}{cc}
\hline characteristic & parameter \\
\hline$R / m m$ & $200 \sqrt{3} / 3$ \\
$r / m m$ & 40 \\
$n / m m$ & 120 \\
$S / m m$ & 240 \\
$\mathrm{~m} / \mathrm{mm}$ & 200 \\
$\mathrm{e} / \mathrm{mm}$ & 40 \\
$\mathrm{~h} / \mathrm{mm}$ & 40 \\
$\mathrm{~L} / \mathrm{mm}$ & 75 \\
point $A$ - Coordinate $/ \mathrm{mm}$ & $0,75,-317$ \\
G/N & 10
\end{tabular}

By the equation (5) (8) can be solved $\omega_{i}, i=1,2,3$.

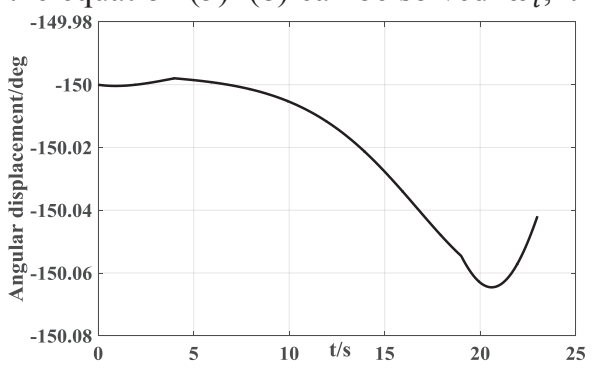

Figure 3. The angular displacement of the first drive joint

General model Fourier4: $\omega_{1}=\mathrm{a} 0+\mathrm{a} 1 * \cos (\mathrm{t} * \mathrm{w})+$ $\mathrm{b} 1 * \sin (\mathrm{t} * \mathrm{w})+\mathrm{a} 2 * \cos (2 * \mathrm{t} * \mathrm{w})+\mathrm{b} 2 * \sin (2 * \mathrm{t} * \mathrm{w})+\mathrm{a} 3 * \cos (3 * \mathrm{t} *$ $\mathrm{w})+\mathrm{b} 3 * \sin (3 * \mathrm{t} * \mathrm{w})+\mathrm{a} 4 * \cos (4 * \mathrm{t} * \mathrm{w})+\mathrm{b} 4 * \sin (4 * \mathrm{t} * \mathrm{w})$

Coefficients (with $95 \%$ confidence bounds): $\mathrm{a} 0=-150 ; \quad \mathrm{a} 1=0.03526 ; \quad \mathrm{b} 1=-0.11 ; \quad \mathrm{a} 2=-0.08551 ; \quad \mathrm{b} 2=$ $-0.01198 ; \mathrm{a} 3=-0.003307 ; \mathrm{b} 3=0.0354 ; \mathrm{a} 4=0.007745$; b4 $=0.001049 ; \mathrm{w}=0.1545$.

Goodness of fit: SSE: 0.0001122, R-square: 0.9996, Adjusted R-square: 0.9996, RMSE: 0.0004453.

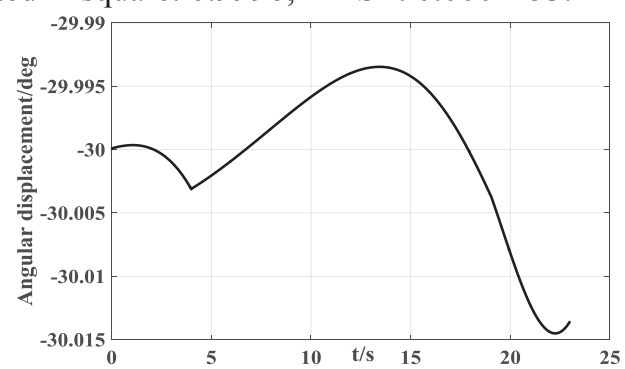

Figure 4. The angular displacement of the second drive joint 
General model Fourier4: $\omega_{2}=\mathrm{a} 0+\mathrm{a} 1 * \cos (\mathrm{t} * \mathrm{w})+$ $\mathrm{b} 1 * \sin (\mathrm{t} * \mathrm{w})+\mathrm{a} 2 * \cos (2 * \mathrm{t} * \mathrm{w})+\mathrm{b} 2 * \sin (2 * \mathrm{t} * \mathrm{w})+\mathrm{a} 3 * \cos (3 * \mathrm{t} *$ $\mathrm{w})+\mathrm{b} 3 * \sin (3 * \mathrm{t} * \mathrm{w})+\mathrm{a} 4 * \cos (4 * \mathrm{t} * \mathrm{w})+\mathrm{b} 4 * \sin (4 * \mathrm{t} * \mathrm{w})$

Coefficients (with $95 \%$ confidence bounds): $\mathrm{a} 0=-2351 ; \mathrm{a} 1=3230 ; \mathrm{b} 1=2015 ; \mathrm{a} 2=-927.6 ; \mathrm{b} 2=-1819$; $\mathrm{a} 3=-28.86 ; \mathrm{b} 3=646.6 ; \mathrm{a} 4=46.57 ; \mathrm{b} 4=-78.94 ; \mathrm{w}=0.03169$.

Goodness of fit: SSE: 3.142e-05, R-square: 0.9984, Adjusted R-square: 0.9984, RMSE: 0.0002356.

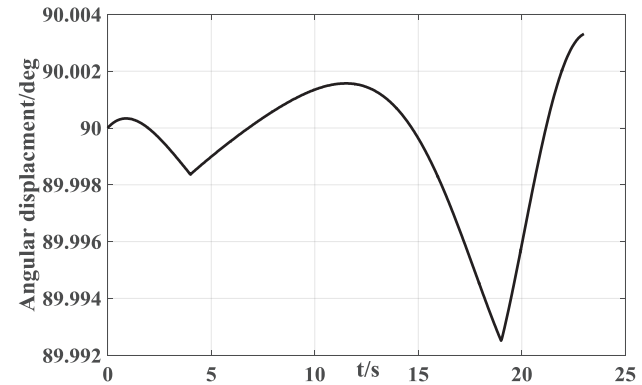

Figure 5 The angular displacement of the third drive joint

General model Fourier4: $\omega_{3}=\mathrm{a} 0+\mathrm{a} 1 * \cos (\mathrm{t} * \mathrm{w})+$ $\mathrm{b} 1 * \sin (\mathrm{t} * \mathrm{w})+\mathrm{a} 2 * \cos (2 * \mathrm{t} * \mathrm{w})+\mathrm{b} 2 * \sin (2 * \mathrm{t} * \mathrm{w})+\mathrm{a} 3 * \cos (3 * \mathrm{t} *$ $w)+b 3 * \sin (3 * t * w)+a 4 * \cos (4 * t * w)+b 4 * \sin (4 * t * w)$.

Coefficients (with $95 \%$ confidence bounds): $\mathrm{a} 0=87.41 ; \mathrm{a} 1=2.052 ; \mathrm{b} 1=3.798 ; \mathrm{a} 2=1.332 ; \mathrm{b} 2=-2.052$; $\mathrm{a} 3=-0.8618 ; \mathrm{b} 3=-0.05547 ; \mathrm{a} 4=0.06522 ; \mathrm{b} 4=0.128 ; \mathrm{w}=0.096$ 55.

Goodness of fit: SSE: 6.305e-05, R-square: 0.9806, Adjusted R-square: 0.9803, RMSE: 0.0003338 .

The angular displacement-time curves of Fig.3 to Fig. 5 as the control are added to the robot drive joint. The angular velocity of the robot-driven joint can be solved as follows Fig. 6 to Fig. 8

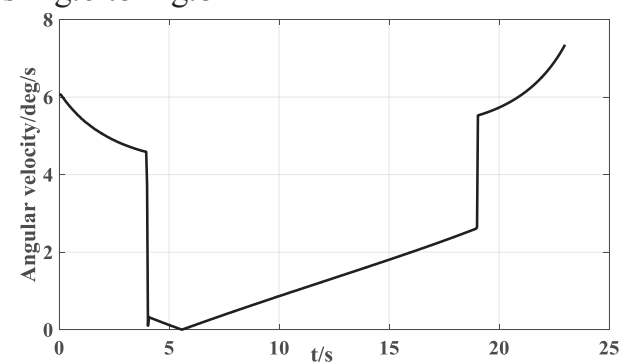

Figure 6. The angular velocity of the first drive joint

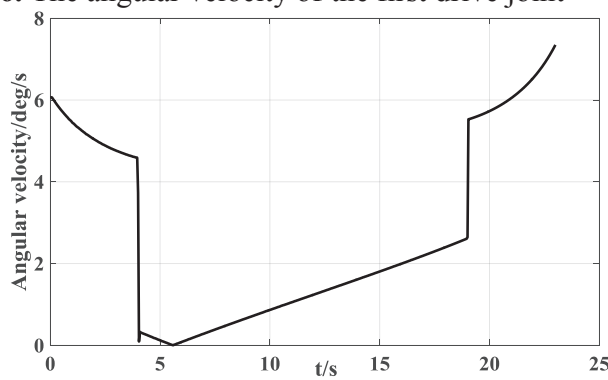

Figure 7. The angular velocity of the second drive joint

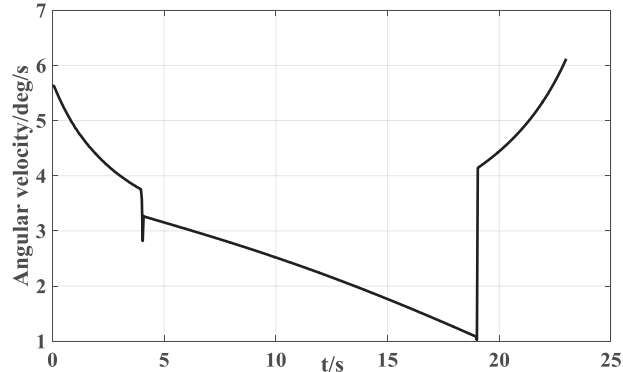

Figure 8. The angular velocity of the third drive joint

The door-shaped curve as the trajectory of the Delta robot pick-and -place operation will give the drive joints to bring the rigid impact.

From Fig. 6 to Fig. 8, it can be seen that when the crank-slider mechanism is applied to the Delta robot pick-and-place operation, the angular velocities of the three driving joints suddenly change at $3.55 \mathrm{~s}$ and $19.05 \mathrm{~s}$. Although the rigid impact under this working condition of the pick-and-place curve of the door-shaped has not been eliminated yet.

\section{Solve the driving joint's force and torque}

\subsection{Forms a crank-slider at the drive joint}

Assuming that the load of the moving platform in the Delta parallel robot pick-up operation is $G$, the force of the three branches on the moving platform is shown in Fig.9, the force of the fixed platform is shown in Fig.10.

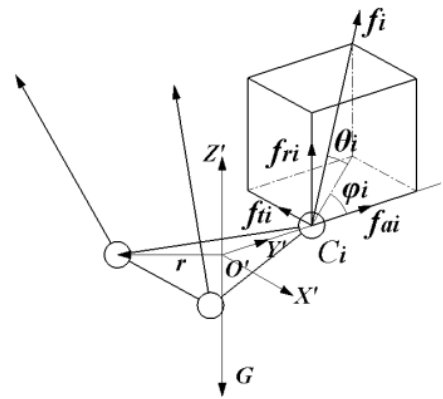

Figure 9. Force diagram of moving platform

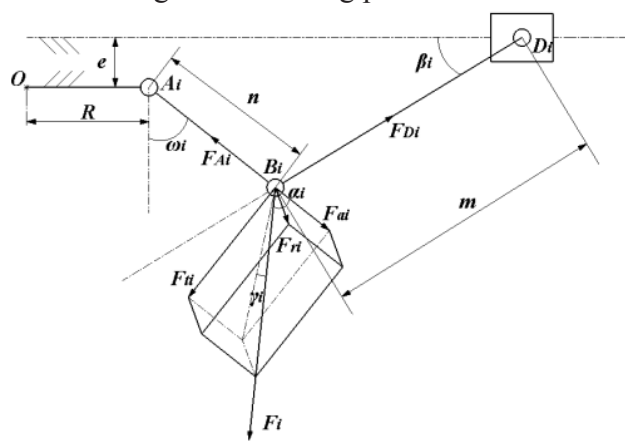

Figure 10. Force diagram of fixed platform

The force and the torque of the drive joints can be solved by using the Fig. 9 and Fig. 10, as the following equation $(9) \sim(11)$. 


$$
\begin{gathered}
F_{A i}=F_{i} \cos \gamma_{i} \cos \alpha_{i}+\frac{F_{i} \cos \gamma_{i} \sin \alpha_{i}}{\cos \left(\omega_{i}-\beta_{i}\right)} \sin \left(\omega_{i}-\beta_{i}\right) \\
\beta_{i}=\tan ^{-1} \frac{e+m \cos \omega_{i}}{n} \\
M_{i}=F_{A i} m \sin \gamma_{i}
\end{gathered}
$$

\subsection{Don't form a crank-slider at the drive joint}

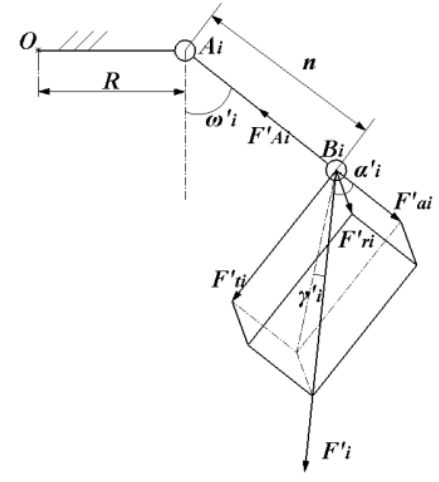

Figure 11. Force diagram of fixed platform

The force and the torque of the drive joints can be solved by using the Fig. 9 and Fig. 11, as the following equation $(12) \sim(13)$.

$$
M_{i}^{\prime}=\sqrt{\left(m F_{i}^{\prime} \sin \gamma_{i}^{\prime}\right)^{2}+\left(m F_{i}^{\prime} \cos \gamma_{i}^{\prime} \sin \gamma_{i}^{\prime}\right)^{2}}
$$

\subsection{The effect of crank-slider on the driving}

(1) On the impact of the force on the drive joints

$$
\Delta F_{A i}=F_{i} \sin \gamma_{i} \cdot \frac{\tan \omega_{i}-\frac{e+m \cos \omega_{i}}{n}}{1+\frac{\tan \omega_{i}\left(e+m \cos \omega_{i}\right)}{n}}
$$

$\left|\omega_{i}\right|,\left|\gamma_{\mathrm{i}}\right| \in(0, \pi / 2)$.

If $\Delta \mathrm{F}_{\mathrm{Ai}}<0$ is less than zero, the Delta robot that drive joints forms a crank-slider mechanism will have less force on the drive joint during the pick-and-place operation.

(2) On the impact of the output coupling torque of the joint

$$
\Delta M_{i}=M_{i}-M_{i}^{\prime}
$$

Through the formulas (11) and (13) we can draw the conclusion: the Delta robot that drive joints forms a crank-slider mechanism will have smaller output torqut on the drive joint during the pick-and-place operation.

\section{Solve the displacement changes of sliders}

According to the kinematics inverse solution theory of Delta robot, solve the linear displacement of the slider linked at the drive joints, as follows Fig.12 to Fig.14.

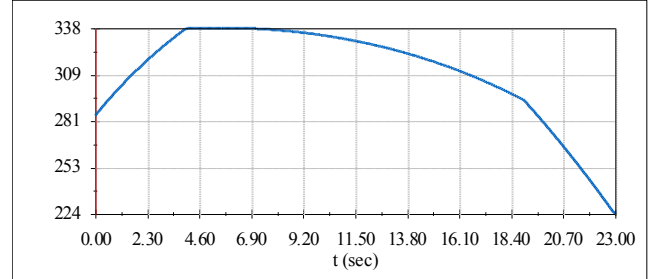

Figure 12. The linear displacement of the first slider

General model Fourier2: $\quad \mathrm{S} 1=\mathrm{a} 0+\mathrm{a} 1 * \cos \left(\mathrm{t}^{*} \mathrm{w}\right)+$ $\mathrm{b} 1 * \sin (\mathrm{t} * \mathrm{w})+\mathrm{a} 2 * \cos (2 * \mathrm{t} * \mathrm{w})+\mathrm{b} 2 * \sin (2 * t * \mathrm{w})$.

Coefficients (with $95 \%$ confidence bounds):

a0 $=182.3 ; \quad$ a1 $=54.41 ; \quad$ b1 $=190.1 ; \quad$ a2 $=46.32 ; \quad$ b $2=$ $-15.93 ; \mathrm{w}=0.1264$

Goodness of fit: SSE: 924.6, R-square:0.998; Adjusted R-square: 0.998, RMSE: 1.274.

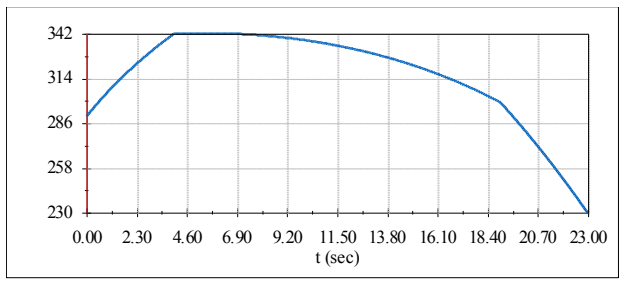

Figure 13. The linear displacement of the second slider

General model Fourier2: $\quad \mathrm{S} 2=\mathrm{a} 0+\mathrm{a} 1 * \cos (\mathrm{t} * \mathrm{w})+$ $\mathrm{b} 1 * \sin (\mathrm{t} * \mathrm{w})+\mathrm{a} 2 * \cos (2 * \mathrm{t} * \mathrm{w})+\mathrm{b} 2 * \sin (2 * \mathrm{t} * \mathrm{w})$.

Coefficients (with $95 \%$ confidence bounds):

a0 $=168.4 ; \quad$ a $1=70.78 ; \quad b 1=210.9 ; \quad$ a $2=49.67 ; \quad$ b2 $=$ $-23.73 ; \mathrm{w}=0.1214$.

Goodness of fit: SSE: 870.6, R-square: 0.9981, Adjusted R-square: 0.9981, RMSE: 1.236.

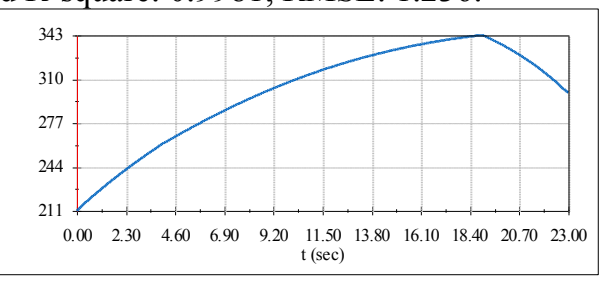

Figure 14. The linear displacement of the third slider

General model Fourier2: $\mathrm{S} 3=\mathrm{a} 0+\mathrm{a} 1 * \cos \left(\mathrm{t}^{*} \mathrm{w}\right)+$ $\mathrm{b} 1 * \sin (\mathrm{t} * \mathrm{w})+\mathrm{a} 2 * \cos (2 * t * \mathrm{w})+\mathrm{b} 2 * \sin (2 * t * \mathrm{w})$.

Coefficients (with $95 \%$ confidence bounds): $\mathrm{a} 0=270.3 ; \mathrm{a} 1=-63.72 ; \quad \mathrm{b} 1=37.54 ; \quad \mathrm{a} 2=6.176 ; \quad \mathrm{b} 2=$ $14.68 ; \mathrm{w}=0.172$.

Goodness of fit: SSE: 971.6; R-square: 0.9987; Adjusted R-square: 0.9987; RMSE: 1.306.

Solving the linear displacement of the slider that linked at the drive joints, it facilitates use to linear robot and rotary motor to coordinated control the Delta robot that performs the pick-and-place operations.

\section{Conclusion}

By absorbing the advantages of the rotary-driven Delta robot and linear-driven Delta robot, a Delta robot for pick-and-place operation that forms a crank-slider at the drive joint is designed. To take the most common gate 
shaped curve in Cartesian space as the motion trail of robotic pick-and-place operation, from many aspects of analysis that application value of slider-crank mechanism in pick-and-place operation of Delta robot.

(1) The angular velocity of during the pickand place operation has been solved. The crank-slider mechanism is applied to the Delta robot for pick-and-place operation, although the rigid impact under this working condition of the pick-and-place curve of the door-shaped has not been eliminated yet.

(2) The Delta robot that drive joints forms a crank-slider mechanism will have smaller force and output torqut on the drive joint during the pick-and-place operation.

(3) According to the kinematics inverse solution theory of Delta robot, solve the linear displacement of the slider linked at the drive joints, it provides a reference for Delta robot which uses rotary motor and linear motor to collaborative control the operation of pick-and-place.

\section{References}

1. CLAVELR. Device for the movement and positioning of an element in space, Europe: EP0250470 B1[P]. 1991-07-17.

2. Brogardh T. Present and future robot control development - An industrial perspective[J]. Annual Reviews in Control, 2006, 31(1): 69-79.

3. Poppeova V, Uricek J, Bulej V, et al. Delta robots Robots for high speed manipulation[J]. Tehnicki Vjesnik, 2011, 18(3):435-445.

4. Bouri M, Clavel R. The linear Delta: Development and application[C] // The 41st International Symposium on Robotics. Frankfurt, Germany: VDE, 2010: 1198-1205.

5. Milutinovic D, Slavkovic N, Kokotovic B, et al. Kinematic modeling of reconfigurable parallel robots based on Delta concept[J]. Journal of Production Engineering, 2012, 15(2): 71-74.

6. Bonev I. Delta paraller robot- The story of success[EB/OL]. (2001-05-06) [2013-06-29]. http://www.parallemic.org/ Review/Review002. html.
7. Pierrot F, Nabat V, Company O, et al. Optimal design of a 4-DOF parallel manipulator: From academia to industry[J]. IEEE Transactions on Robotics, 2009, 25(2): 213-224.

8. Pierrot F, Marquet F, Company O, et al. H4 parallel robot: modeling, design and preliminary experiments[C]//Robotics and Automation, 2001. Proceedings 2001 ICRA. IEEE International Conference on. IEEE, 2001, 4: 3256-3261.

9. Nabat V, de la O RODRIGUEZ M, Company O, et al. Par4: Very high speed parallel robot for pick-and-place[C]//Intelligent Robots and Systems, 2005.(IROS 2005). 2005 IEEE/RSJ International Conference on. IEEE, 2005: 553-558.

10. Liu S, Huang T, Mei J, et al. Optimal design of a 4-DOF SCARA type parallel robot using dynamic performance indices and angular constraints[J]. Journal of Mechanisms and Robotics, 2012, 4(3): 031005.

11. ZHANG Limin, MEI jiangping, HUANG Tian. Dynamic Dimensional Synthesis of Delta Robot[J]. Chinese Journal of Mechanical Engineering, 2010, 46(3): 2-3.

12. Codourey A. Dynamic modeling of parallel robots for computed-torque control implementation[J]. The International Journal of Robotics Research, 1998, 17(12): 1325-1336.

13. WANG P, MEI J, HUANG T. Time Optimal Path Planning of a 2-DOF High-Speed Parallel Robot [J]. Journal of Tianjin University, 2007, 10: 000.

14. Bouri M, Clavel R. The linear Delta: Developments and applications[C] // The 41st International Symposium on Robotics. Frankfurt, Germany: VDE, 2010: 1198-1205.

15. Milutinovic D, Slavkovic N, Kokotovic B, et al. Kinematic modeling of reconfigurable parallel robots based on Delta concept[J]. Journal of Production Engineering, 2012, 15(2): 71-74.

16. Xie zexiao, Shang Dawei, Ren Ping. Optimization and Experimental Verification of Pick-and-place Trajectory for a Delta Parallel Robot Based on Lame Curves[J]. Chinese Joumal of Mechanical Engineering, 2015, 51(1): 52-55. 